\title{
Boron-Ate Complexes as Chiral Nucleophiles for Asymmetric Synthesis
}
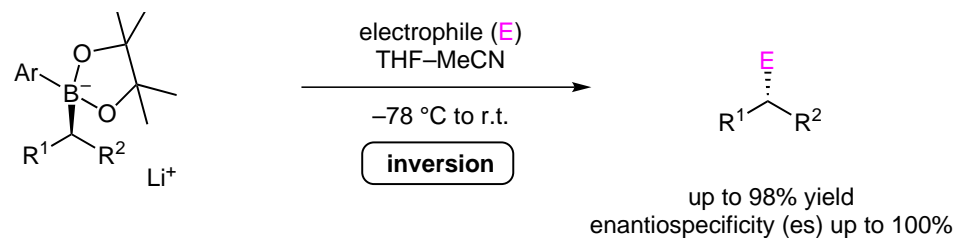

$\mathrm{Ar}=4-\mathrm{MeOC}_{6} \mathrm{H}_{4}, 3,5-\left(\mathrm{CF}_{3}\right)_{2} \mathrm{C}_{6} \mathrm{H}_{3}$

$\mathrm{R}^{1}=\mathrm{Ar}$, Ar-substituted Alk

$\mathrm{R}^{2}=\mathrm{Me}, \mathrm{Et}, i-\mathrm{Bu}$, allyl, substituted Alk

$\mathrm{E}=$ various electrophiles

Selected examples:

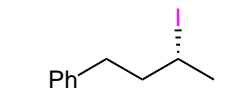

$80 \%$ yield

$100 \%$ es

$\mathrm{E}=\mathrm{N}$-iodosuccinimide

$\mathrm{Ar}=4-\mathrm{MeOC}_{6} \mathrm{H}_{4}$<smiles>CC(c1ccccc1)C1C=CC=CC=C1</smiles>

$98 \%$ yield

$100 \%$ es

$\mathrm{E}=$ tropylium tetrafluoroborate

$\mathrm{Ar}=4-\mathrm{MeOC}_{6} \mathrm{H}_{4}$
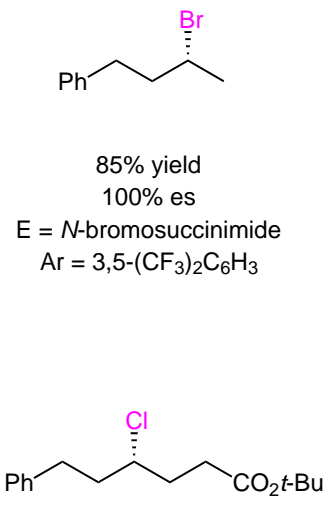

$84 \%$ yield

$100 \%$ es

$\mathrm{E}=$ trichloroisocyanuric acid

$\mathrm{Ar}=3,5-\left(\mathrm{CF}_{3}\right)_{2} \mathrm{C}_{6} \mathrm{H}_{3}$

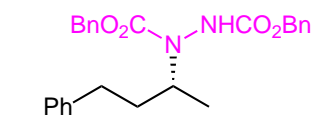

$66 \%$ yield

$92 \%$ es

$\mathrm{E}=$ dibenzyl azodicarboxylate

$\mathrm{Ar}=3,5-\left(\mathrm{CF}_{3}\right)_{2} \mathrm{C}_{6} \mathrm{H}_{3}$

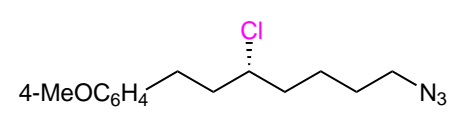

$$
\begin{gathered}
61 \% \text { yield } \\
98 \% \text { es } \\
\mathrm{E}=\text { trichloroisocyanuric acid } \\
\mathrm{Ar}=3,5-\left(\mathrm{CF}_{3}\right)_{2} \mathrm{C}_{6} \mathrm{H}_{3}
\end{gathered}
$$

Significance: The authors report that secondary chiral boronic esters can be converted into reactive nucleophiles by addition of an aryllithium reagent. These enantiomerically enriched nucleophiles react with a broad range of electrophiles with inversion of stereochemistry.
Comment: By changing the substituents on the aryl group on boron, a switch in mechanism from a classical $2 \mathrm{e}^{-}$pathway (nucleophilic substitution) to a radical pathway was observed. Therefore, electron-poor boronic esters favor the desired nucleophilic substitution, whereas electron-rich esters give racemized products. 